\title{
Real-time feedback as a method of monitoring walking velocity during gait analysis
}

\author{
Sarah T. Ridge \\ Brigham Young University - Provo, sarah_ridge@byu.edu \\ James G. Richards
}

Follow this and additional works at: https://scholarsarchive.byu.edu/facpub

Part of the Exercise Science Commons

\section{Original Publication Citation}

Ridge, S, Richards, J. (2011) Real-time Feedback as a Method of Monitoring Walking Velocity during Gait Analysis. Gait and Posture, 34(4), 564-566.

\section{BYU ScholarsArchive Citation}

Ridge, Sarah T. and Richards, James G., "Real-time feedback as a method of monitoring walking velocity during gait analysis" (2011). Faculty Publications. 2017.

https://scholarsarchive.byu.edu/facpub/2017 


\title{
Real-time feedback as a method of monitoring walking velocity during gait analysis
}

Sarah TragerRidge, James G.Richards

\begin{abstract}
When quantifying the mechanics of gait, it is important to ensure that subjects maintain a consistent walking velocity during gait analysis trials. Most methods of measuring walking velocity do not produce data until after the subject has completed the trial. This often results in discarding completed trials from analysis because the subject's velocity was not within an acceptable range. Real-time feedback of position data can be used to help subjects adjust their walking velocity during the trial, when necessary. Results from 14 subjects who participated in gait analysis using real-time feedback to monitor their walking velocity show that they were able to stay within an acceptable range of their target walking velocities (each subject's preferred velocity and $150 \%$ of their preferred velocity) during $90 \%$ and $80 \%$ of trials, respectively. This method allows for accurate and efficient data collections without the use of additional equipment.
\end{abstract}

\section{Keywords}

Gait, Walking Velocity, Real-time feedback

\section{Introduction}

Monitoring a subject's walking or running velocity is important when performing gait analysis. Since previous research has shown that walking mechanics (joint angles, moments, powers, EMG activity, and temporal-spatial characteristics) change at different walking velocities, researchers often require a subject to maintain a certain walking or running pace during data collections [1-3]. However, it is often difficult for subjects to control their velocity with the precision required, especially when walking faster or slower than their own preferred velocity. Typical methods of calculating over-ground walking velocity during data collections include the use of a timer triggered by photocells set a pre-determined distance apart, using a stopwatch as subjects walk a pre-determined distance, asking subjects to walk to the beat of a metronome, and/or having subjects walk on a treadmill [4-6]. Unfortunately, other than using a treadmill (which may not be appropriate for some studies) these methods do not provide velocity data until after the trial is completed, which often leads to a need for repeated trials after instructing the subject to walk “a little faster" or "a little slower". Therefore, using real-time visual feedback to monitor walking velocity during data collection may result in a more efficient testing environment by reducing the number of trials needed to obtain quality data from a subject. 
Real-time feedback has been used during biomechanical analyses for purposes such as helping participants alter their mechanics to avoid injury, match a certain protocol, and give information about force production or muscle activation [7-10]. As a method of monitoring walking velocity, real-time feedback allows for consistent walking velocities, potentially reducing the need to perform trials that may ultimately be discarded from data analysis. More importantly, this should allow for shorter duration data collections, which is crucial when analyzing children and/or patients for whom walking is difficult. This method can enhance the data collection process in research using marker-based video motion analysis.

\section{Methods}

Data were collected from 14 healthy children between the ages of 11 and 17 years (average age $14.3 \pm 2.1$ years). Gait analysis was performed using 10 Eagle Digital Motion Analysis Cameras (Motion Analysis Corporation, Santa Rosa, CA) collecting at $60 \mathrm{~Hz}$. Forty-one reflective markers were applied to anatomical landmarks to create 12 segments for full body analysis. Marker position data was streamed in real-time from the motion analysis software to custom LabVIEW software (National Instruments, Austin, TX).

Subjects walked across a designated walkway 3-5 times to establish their preferred walking velocities. Average walking velocity was collected throughout each trial, and trials were averaged to determine the preferred walking speed. In real time, average walking velocity was represented by the average velocity of the sacral marker along the axis corresponding to the direction of walking. The velocity of the sacral marker was calculated during each trial by dividing the change in marker position between frames by the time elapsed between frames. Any data that resulted in a velocity greater than $3.0 \mathrm{~m} / \mathrm{s}$ was discarded, as this was considered erroneous data. This ensured data was not included from before or after the edges of the data collection volume or from other frames in which there was no data for the sacral marker position. Calculation of the change in position from a frame with no data to a frame with data resulted in artificially high velocities.

Using this information, subjects performed the rest of the walking trials while monitoring their position on a screen at the end of the walkway (Fig. 1). The top slider represented the location of the sacral marker in the direction of the walk as the subject moved through the volume. The bottom slider represented the subject's instantaneous target position along the walkway, based on his/her start position in the volume and his/her preferred walking speed. The sliders aligned when the subject entered the data collection volume at which point the bottom slider progressed from left to right at the subject's preferred walking speed. The subject was instructed to keep the top slider aligned with the bottom slider. Trials were also performed at $150 \%$ of the subject's preferred speed, with the bottom slider reflecting the faster speed. 


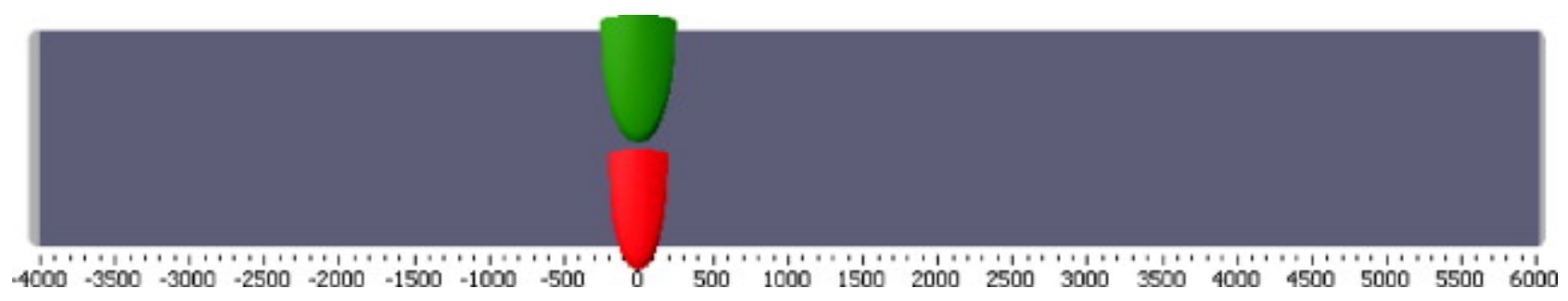

Fig. 1. Image projected onto the screen at the end of the walkway. The numbers along the bottom represented the $x$-axis of the laboratory coordinate system (subjects always walked in the $+x$ direction).

To determine the efficacy of this method at monitoring walking speed, the percent difference between each subject's target speed and his/her actual average speed throughout the trial were calculated and compared.

The data reported in this paper were collected as part of a protocol designed to investigate strategies for gait termination. Subjects were required to stop walking as soon as they saw a stop sign appear on the same screen on which they were monitoring their velocity. Subjects did not know when this signal would appear. As a result, some subjects were slowing slightly in anticipation of the stop sign as they reached the end of the volume. Due to the nature of the task under investigation (unplanned gait termination), it was crucial that we were able to monitor subjects' walking velocities throughout the trial. Walking velocities presented in the paper were calculated from the moment subjects entered the data collection volume until the "stop" signal was presented.

\section{Results}

Subjects were able to maintain their preferred speed by visually monitoring their own velocities during $90.6 \%$ of the trials. The fast condition was more difficult, resulting in subjects maintaining their faster speed during $82 \%$ of the trials, though seven subjects were within $\pm 10 \%$ in every trial. Table 1 lists the total number of trials completed by each subject and the number of those trials completed with an average velocity within $\pm 10 \%$ of his/her target velocity.

Table 1. Number of walking trials completed by each subject and the number of trials in which the subject's actual walking velocity was within $\pm 10 \%$ of the target walking velocity.

\begin{tabular}{lllll}
\hline Subject & \# Trials & \# Within $\mathbf{\pm 1 0 \%}$ & \# Trials & \# Within $\mathbf{\pm 1 0 \%}$ \\
1 & 8 & 8 & 6 & 6 \\
2 & 8 & 5 & 12 & 8
\end{tabular}




\begin{tabular}{|c|c|c|c|c|}
\hline Subject & \# Trials & \# Within $\pm 10 \%$ & \# Trials & \# Within $\pm \mathbf{1 0} \%$ \\
\hline 3 & 10 & 10 & 6 & 3 \\
\hline 4 & 10 & 9 & 10 & 4 \\
\hline 5 & 10 & 8 & 7 & 4 \\
\hline 6 & 5 & 4 & 17 & 17 \\
\hline 7 & 10 & 9 & 10 & 10 \\
\hline 8 & 12 & 10 & 6 & 6 \\
\hline 9 & 9 & 9 & 11 & 11 \\
\hline 10 & 9 & 8 & 8 & 6 \\
\hline 11 & 10 & 10 & 15 & 12 \\
\hline 12 & 16 & 16 & 9 & 9 \\
\hline 13 & 16 & 15 & 14 & 14 \\
\hline 14 & 17 & 17 & 12 & 10 \\
\hline
\end{tabular}

Velocity data from a representative trial is shown in Fig. 2. The steep decline in velocity at the end of the graph shows data after the subject was given the signal to stop. Despite the challenge presented by this stopping protocol, subjects maintained a speed that was similar to their target speed throughout the trial. 


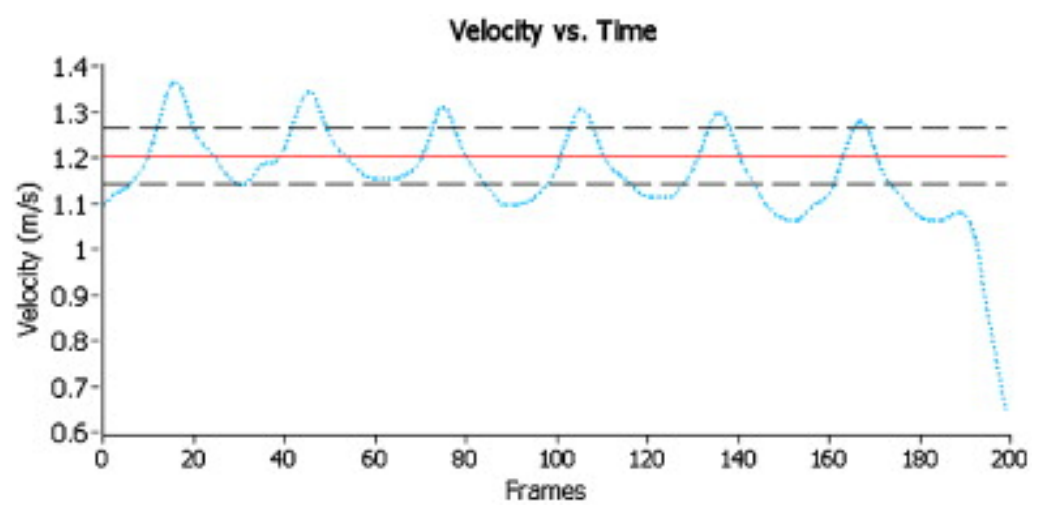

Fig. 2. Graph of a representative subject's velocity throughout one walking trial. The dashed blue line represents the frame-by-frame velocity calculated from the position of the sacral marker. The red solid line at approximately $1.2 \mathrm{~m} / \mathrm{s}$ is the subject's preferred walking velocity (or target velocity), while the straight, dashed lines represent $\pm 10 \%$ of her preferred walking velocity. (For interpretation of the references to color in this figure legend, the reader is referred to the web version of the article.)

\section{Discussion}

Subjects in this study were effective at staying in an acceptable range of their target velocity using a real-time visual feedback method of monitoring walking velocity. In addition to the added efficiency during data collection, monitoring velocity data in real-time also allows researchers and clinicians to see changes in velocity throughout the trial, rather than just an average for the whole trial. These changes in walking velocity may be important to note during the data collection.

There are some limitations to this method of monitoring walking velocity. The data collection volume must be large enough to allow the subject to be able to adjust his/her walking speed and the projection must be clearly visible to subjects throughout the trial. However, based on this study, monitoring walking velocity in real-time may be helpful for increasing the efficiency of data collections involving walking or running protocols when controlling speed is important, but a treadmill is not appropriate.

\section{Conclusion}

Using position data to monitor walking velocity in real time offers an effective way of allowing subjects to monitor their walking velocity throughout a trial and should result in fewer trials, which may make gait analysis easier on the subject. During motion analysis, when marker data is already being collected, this method requires little extra hardware and provides immediate feedback to the subject and the researcher.

\section{Conflict of interest statement}

There are no conflicts of interest or acknowledgements to report. 


\section{References}

[1] Murray MP, Mollinger LA, Gardner GM, Sepic SB. Kinematic and EMG patterns during slow, free, and fast walking. J Orthop Res 1984;2(3):272-80.

[2] Schwartz MH, Rozumalski A, Trost JP. The effect of walking speed on the gait of typically developing children. J Biomech 2008;41(8):1639-50.

[3] Stansfield BW, Hillman SJ, Hazlewood ME, Lawson AA, Mann AM, Loudon IR, et al. Sagittal joint kinematics, moments, and powers are predominantly characterized by speed of progression, not age, in normal children. J Pediatr Orthop 2001;21(3):403-11.

[4] Bishop M, Brunt D, Pathare N, Patel B. The effect of velocity on the strategies used during gait termination. Gait Posture 2004;20(2):134-9.

[5] Robbins SM, Maly MR. The effect of gait speed on the knee adduction moment depends on waveform summary measures. Gait Posture 2009;30(4):543-6.

[6] Fey NP, Silverman AK, Neptune RR. The influence of increasing steady-state walking speed on muscle activity in below-knee amputees. J Electromyogr Kinesiol 2010;20(1):155-61.

[7] Koritnik T, Koenig A, Bajd T, Riener R, Munih M. Comparison of visual and haptic feedback during training of lower extremities. Gait Posture 2010;32(4):540-6.

[8] Noehren B, Scholz J, Davis I. The effect of real-time gait retraining on hip kinematics, pain and function in subjects with patellofemoral pain syndrome. Br J Sports Med 2011;45(9):691-6.

[9] Rice I, Gagnon D, Gallagher J, Boninger M. Hand rim wheelchair propulsion training using biomechanical real-time visual feedback based on motor learning theory principles. J Spinal Cord Med 2010;33(1):33-42.

[10] Wheeler JW, Shull PB, Besier TF. Real-time knee adduction moment feedback for gait retraining through visual and tactile displays. J Biomech Eng 2011;133(4):041007. 